\title{
Traffic Offloading in 5G Heterogeneous Networks using Rank based Network Selection
}

\author{
Anand Gachhadar ${ }^{1}$, Faizan Qamar ${ }^{2}$, Dhawa Sang Dong ${ }^{1}$, Mohammed B. Majed ${ }^{3}$, Effariza Hanafi ${ }^{2}$ and \\ Iraj Sadegh Amiri ${ }^{4,5, *}$
}

\begin{abstract}
${ }^{I}$ Department of Electrical and Electronic Engineering, School of Engineering, Kathmandu University, Dhulikhel, Nepal. ${ }^{2}$ Department of Electrical Engineering, Faculty of Engineering, University of Malaya, 50603, Kuala Lumpur, Malaysia. ${ }^{3}$ College of Science and Technology, University of Human Development (UHD), Assulaymaniyah, KRG, Iraq. ${ }^{4}$ Computational Optics Research Group, Ton Duc Thang University, 700000, Ho Chi Minh City, Vietnam. ${ }^{5}$ Faculty of Applied Sciences, Ton Duc Thang University, 700000, Ho Chi Minh City, Vietnam.
\end{abstract}

\begin{abstract}
The exponential growth of mobile data traffic and a limited number of spectrum resources has been a big challenge for cellular network providers, henceforth traffic offloading has become one of the most critical issues especially in $5 \mathrm{G}$ Heterogeneous Networks (HetNets). Further, network selection plays a vital role for traffic offloading in a cellular network to maintain Quality of Service (QoS), increasing offloading efficiency and throughput. In order to efficiently utilize spectral resources, a Technique for Order Preference by Similarity to Ideal Solution (TOPSIS) algorithm is proposed to be used for ranking a candidate network. The proposed algorithm helps in alleviating the spectrum shortage by offloading the data traffic over Wi-Fi network using unlicensed spectrum. In this work, analysis of the performance of the proposed system model through simulation of an analytical framework has been made. The results have been accumulated in terms of cumulative handover, throughput, the extent of equilibrium \& offloading efficiency with respect to residence time and the number of Wi-Fi Access Points (AP's). Analysis proves that the proposed algorithm improves the equilibrium extent and throughput as compared to traditional Load balancing (LB) and SDN based LB mechanisms. It also shows that offloading efficiency is highly improved over Wi-Fi density and residence time.
\end{abstract}

Keywords: Heterogeneous Networks; Traffic Offloading; Rank based Network Selection; Extent of equilibrium; Off-loading efficiency

\section{Introduction}

Over the last decade, the advancements in wireless technology have increased exponentially. This growth is very much a consequence of rapid technological developments and ever-so increasing user demands. As a matter of fact, specifically from communication perspective, a lot of technological developments have been made recently and are in progress to cater the ever so increasing user demands, service requirements and quality of experience, such as, with the use of high-frequency mm-wave bands [13], Device to Device Communication (D2D) [4], Cooperative and Heterogeneous Network (HetNet) [5, 6], Coordinated Multipoint (CoMP) [7], Internet of things (IoT) [8], Carrier Aggregation (CA) [9], Cognitive Radio (CR) [10], Passive Optical Network (PON) [11], Massive Multiple Input Multiple Output (MIMO) [12], NonOrthogonal Multiple Access (NOMA) [13], Mobile Ad-hoc Network (MANET) [14], Vehicular ad-hoc networks (VANET) [15], Fog Computing [16]. Further, 5G will also be capable of incorporating existing technologies such as several advance power optimizations techniques $[17,18]$ and

*E-mail address: irajamiri2018@gmail.com ISSN: $1791-2377$ @ 2019 Eastern Macedonia and Thrace Institute of Technology. All rights reserved. doi:10.25103/jestr.122.02 optimal scheduling algorithms [19] required to cope with user demands meanwhile providing technology beyond fingertips. According to a study, an average area capacity of $25 \mathrm{~Gb} / \mathrm{s} / \mathrm{km}^{2}$ and 1000 times improvements energy consumptions are expected by 2020 because of an enormous increase in a number of electronic devices to be connected (internet of things-IoT) to the cloud [20]. To deal with high teledensity within a specific coverage space, dense deployment of base stations with lower transmission power, named remote radio heads (RRH), was proposed. Deployments were proposed to be made within the coverage area micro-cell of existing micro-base stations (MBS) comprising HetNet architecture [21]. This could result in a possible conflict between the cellular service providers and users due to a rise in overall service cost. It is a direct consequence of an increase in the overall capital expenditure. Although users will experience higher data rates and quality of service, however, with an increase in the number of base stations, the capital cost of the infrastructure will only be going to rise and that would adversely result in comparatively higher cost of service for the users. For this, there could be a solution to reduce the overall operational cost, that is, energy efficient operation and optimal utilization of limited cellular radio resources i.e. spectrally efficient data transmission. Since radio resources are approaching saturation, data offloading is an alternative to 


\section{Journal of Engineering Science and Technology Review 12 (2) (2019) 9 - 16}

address the exponentially increasing traffic requirements which leads us to optimal utilization of resources [22].

Offloading means usage of resources either computational or spectral from neighboring networks when available. Data offloading should be carried out either to balance the load to the nodes or for efficient utilization of both licensed and unlicensed radio resources [23]. Integration of $\mathrm{Wi}-\mathrm{Fi}$ networks to the cellular networks for data offloading is an example of the utility of unlicensed spectrum assigned to Wi-Fi networks. With Wi-Fi networks using unlicensed spectrum, so there won't be any interference between the signals utilizing the spectrum and the Wi-Fi enabled devices. Therefore, almost all the data offloading research activities are being carried over Wi-Fi networks [24]. Data offloading can be carried over either Wi-Fi networks, Device-to-Device (D2D) networks or over Software-Defined networks depending upon data demands and data type to be offload through other networks $[25,26]$. Though, Software-Defined network could be one of the approaches used as intelligent network configuration for data offloading and as it is also configured by the central controller according to architectural need. Offloading mechanism is usually introduced to the communication model while keeping in view of both the user's perspective of cost minimization and of cellular network operator's perspective of optimal usage of resources, meanwhile effectively fulfilling the user data rate demands in compliance with next-generation wireless technologies [27]. For both the cases, the offloading decision process is crucial without loss of significant quality of user experience and offloading efficiency depends upon offloading problem formation and finding optimal solutions.

\section{Contribution}

In this work, a simple opportunistic model to offload traffic utilizing existing Wi-Fi technology with proper network selection is proposed. In order to rank a candidate, it uses TOPSIS algorithm, which utilizes signal to noise ratio (SNR), received signal strength, network load and available bandwidth as benefit parameters [28, 29]. The entrant network which needs to be offloaded is chosen by a network which has the maximum rank value and the offloaded amount of data depends upon the residence time. If a user is unable to offload the whole amount of data within the designated duration in a $\mathrm{Wi}-\mathrm{Fi}$ range, the remaining amount of data is offloaded through the cellular network. The QoS performance of the network is analyzed based on KPI's such as cumulative handover, throughput, the extent of equilibrium \& offloading efficiency with respect to residence time and a number of Wi-Fi APs. The rest of the paper is organized as follows: In Section 2, related work from the literature is discussed. Section 3 discusses the main contributions presented in this work. The evaluation of the system model for HetNet for offloading is depicted in Section 4. Moreover, the proposed Rank Based Network Selection and traffic offloading using Wi-Fi Algorithm is discussed in Section 4 (A) and 4 (B), respectively. After this, all numerical results and discussion are described and explained in Section 5. Finally, the work is concluded in Section 6.

\section{Related Work}

Recently, various traffic offloading mechanisms based on network selection algorithms have been proposed exhaustively in the literature, especially for SDN. In [30], Duan et al. introduced a SDN based resource management algorithm, where the scheme proposes to offload the data traffic over Wi-Fi network using unlicensed spectrum, thus, saving 5G licensed-band cellular spectral resources. This scheme not only addresses the spectral deficiency but also reduces the network congestion in the cellular network, which is the primary network. This approach was also integrated for partial data offloading considering delay sensitivity of real-time applications, where it considers all the time required to enable SDN and offload the data over the Wi-Fi network. Primarily this scheme intends to save primary network resources without any loss of QoS. Another efficient approach was presented by Aujla et al. in [31], which proposed SDN based data offloading scheme for vehicular networks. The aim of the work was to cope with traffic congestion and high speeds within the existing network while using intelligent decision with priority manager and offloading manager. Authors used Stackelberg's game approach with a single leader and many followers for the selection of appropriate networks for traffic offloading. Similarly, Chen et al. in [32] studied the dynamic resource management scheme, named Enterprise Visor engine. The engine manages the distribution of network resources, which are sliced to virtual sub-networks. Their proposed Enterprise Visor engine uses the Open Flow virtualization protocol, which enhances the network utility by $25.7 \%$ as compared to the basic Open Flow framework.

An approach in [33] studied the energy-aware data offloading method, where energy estimation is performed at User Equipment (UE) before making an offloading request to Base Band Unit (BBU) pool. BBU pool reserves the right to approve the request when a neighboring $\mathrm{AP}$ or $\mathrm{RRH}$ is available with optimal transmission rate radio resources to meet the quality of experience demanded along with minimal energy consumption rate. This scheme is efficient, because of centralized information processing of APs, RRHs and UEs at the centralized unit, that is, BBU pool. Moreover, Jin et al. in [34] presented a user equipment preference-aware network selection algorithm for data offloading. It takes offloading decisions based on criteria; application type i.e. delay tolerant or intolerant. Specifically, network information i.e. application type, energy consumption constraints and expected quality of experience (QoE) were the parameters used for intelligent selection of cells for offloading. Unlike other schemes, where Signal to Interference \& Noise Ratio (SINR), cell load and user requirements like power or delay preferences are key attributes utilized for offloading. Furthermore, Fakhfakh and Hamouda in [35] proposed an adaptive learning algorithm i.e. Q-learning, to learn the past quality of user experience and defined a reward parameter for each user to make an offloading decision. Reward parameter was a function of the load of each candidate's APs, handover time, corresponding gain and SINR. Based on the reward criteria, an optimal action is taken for converged AP's selection and setting-up of offloading strategies. Also, the presented algorithm was efficient with low computational complexity. Similarly, Liu et al in [25] studied the hybrid offloading model, where cellular mobile data was either offloaded over a Wi-Fi network or a D2D network, to minimize the cost of data transmission for delay tolerant mobile data. Authors formulated the offloading decision problem as a Finite Horizon Markov Decision Process (FHMDP), which primarily considers the delay sensitivity of the mobile data. In this hybrid offloading model, D2D offloading was 
observed to be minimal cost transmission scheme for delay tolerant data type.

\section{System Model}

We consider the system model i.e. HetNet, to be consisting of a Macro-cell, low powered Femto-cells, and Wi-Fi APs, as shown in Fig. 1. The macrocell BS's communication is over the $5 \mathrm{G}$ licensed-band whereas, Wi-Fi APs communicate over the unlicensed band. Also, the Wi-Fi APs are distributed randomly within each macrocell. Further, all the network components are connected directly to the HetNet Cloud Radio Access Networks (H-CRAN) controller. It is the fundamental component of the $5 \mathrm{G}$ HetNet model, which enables diversity through the integration of HetNets and CRAN [36]. It inherits the tiered deployment of small and macrocells. The small cells such as femtocells, picocells increase the reuse of the spectrum and help in achieving higher capacity. Furthermore, CRAN consists of digital functional units called $\mathrm{BBU}$ to handle computing workload, signal processing, and resource management.

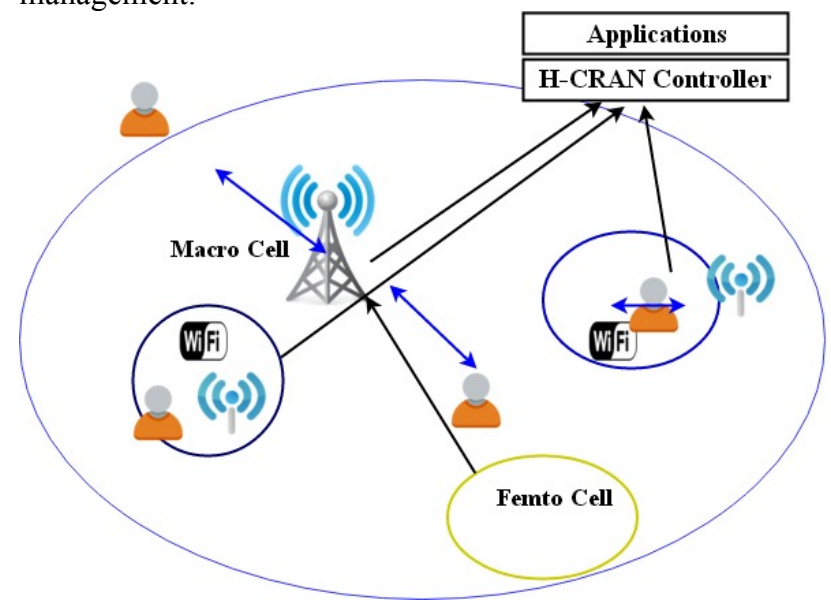

Fig. 1 System Model

\section{A. Rank Based Network Selection Algorithm}

In this section, algorithms for network selection and offloading of cellular traffic through Wi-Fi Access Points (APs) in 5G HetNets are presented. We attempt to alleviate the shortage of spectrum in a densely populated network through communication over the unlicensed band. Offloading will occur whenever the cellular users are within the range of Wi-Fi APs and have $S N R$ greater than the given threshold i.e. $S N R_{t h}$. Further, the equation for received signal strength $R_{x i}$ and $S N R_{i}$ for user $i$ at distance $d$ from the AP with transmission power $P_{t}$ can be written as,

$R_{x i}=P_{t} d^{-\alpha}$

$S N R_{i}=\frac{P_{t} h_{i w} \theta_{i w} L(d)}{I_{i w}}$

where, ' $h_{i w}{ }^{\prime}$ represents the fading power gain, ' $\alpha$ ' represents the path loss exponent, ' $\theta_{i w}{ }^{\prime}$ represents the lognormal shadowing loss and ' $L$ ' represents the miscellaneous losses between user and $\mathrm{AP},{ }^{\prime} I_{i w}{ }^{\prime}$ represents interference from different APs. Also, the average rate ' $r_{i}$ ' of a user at a distance ' $d$ ' can be obtained as

$r_{i}=b_{w} \log _{2}(1+S N R)$
The authors in [37-40] defined load as the number of frames that AP successfully handles per unit time. The successful transmission of the frame within a given time for each user can be calculated as,

$u=\frac{p_{s} T_{s i}}{T_{i d l e}+\bar{T}_{s}+\bar{T}_{C}}$

Where $p_{s}=\tau(1-\tau)^{n-1}$ is a probability that a user transmits successfully, whereas $T_{i d l e}$ is the average idle time, $\bar{T}_{s}$ is the time for successful transmission and $\bar{T}_{c}$ is the time for collision transmission. From [41] the collision probability and transmission probability can be computed as

$\tau=\frac{2\left(1-2 p_{c}\right)}{\left(W_{0}+1\right)\left(1-2 p_{c}\right)+p_{c} W_{0}\left(1-\left(2 p_{c}\right)^{\left.m_{w}\right)}\right.}$

$p_{c}=1-(1-\tau)^{n-1}$

Where ' $\tau$ ' and ' $p_{c}$ ' represents the transmission and collision probability, respectively, ' $W_{0}$ ' is the minimum contention window size, ' $m_{w}$ ' indicates the maximum number of back off-hands and ' $n$ ' represents the number of users within the range of Wi-Fi AP. The load, ' $\delta^{\prime}$, of a WiFi AP can now be calculated as,

$\delta=\sum_{i=1}^{n} u_{i}$

H-CRAN controller handles all the decision, while the network selection for traffic offloading is made using TOPSIS algorithm [42]. Here the candidates for traffic offloading of UE are the Wi-Fi APs and the decision criteria are Bandwidth, Network Load, SNR and RSS. Each decision criteria is weighted, such that $\sum_{j} w_{j}=1$. The H-CRAN controller collects all the traffic information from Wi-Fi AP's cells to calculate the current load on each Wi-Fi APs. The decision matrix is then denoted by,

$D=\left[\begin{array}{c}x_{11} x_{12} x_{13} x_{14} \\ x_{21} x_{22} x_{23} x_{24} \\ \ldots \ldots \ldots \ldots \ldots \ldots \\ \ldots \ldots\end{array} \ldots\right.$

The decision matrix consists of $N$ rows with 4 measurement metrics represented by 4 columns. Where, ' $x_{i j}{ }^{\prime}$ represents the elements of the decision matrix. The weights are assigned by the controller that in this case are assumed to be $W=\left[\begin{array}{llll}0.2 & 0.2 & 0.3 & 0.3\end{array}\right]$. The order preference TOPSIS algorithm requires the following steps:

Step 1: Construct a normalized decision matrix as given by

$r_{i j}=\frac{x_{i j}}{\sqrt{\sum_{i=1}^{N} x_{i j}^{2}}}$

Here ' $N$ ' represents the number of candidate Wi-Fi APs of which the UEs are receiving considerable signal strength.

Step 2: Construct a weighted-normalized decision matrix

$v_{i j}=w_{j} r_{i j}$

Step 3: Determine the positive and negative ideal solutions 
$A^{+}=\left\{v_{1}^{+}, v_{2}^{+}, v_{3,}^{+} v_{4}^{+}\right\}=$ $\left\{\left(\max _{i j} \mid j \in J\right),\left\{\operatorname{minv}_{i j} \mid j \in J^{\prime}\right\}\right\} i=1,2, \ldots . . N, j=$ $1,2,3,4$

$A^{-}=\left\{v_{1}^{-}, v_{2}^{-}, v_{3}^{-}, v_{4}^{-}\right\}=$

$\left\{\left(\operatorname{minv}_{i j} \mid j \in J\right),\left\{\max _{i j} \mid j \in J^{\prime}\right\}\right\} i=1,2, \ldots . . N, j=1,2,3,4$

Here ' $J$ ' is a set of benefit criteria and ' $J$ ' ' is a set of cost criteria, that is, $J=\left\{S N R, R_{x}, b_{w}\right\}$ and $J^{\prime}=\{\delta\}$

Step 4: Calculate the separation measure from positive and negative ideal solutions

$S_{i}^{+}=\sqrt{\sum_{j=1}^{4}\left(v_{i j}-v_{j}^{+}\right)^{2}}$

$S_{i}^{-}=\sqrt{\sum_{j=1}^{4}\left(v_{i j}-v_{j}^{-}\right)^{2}}$

Step 5: Measure the relative closeness to the ideal solution

$$
C_{i}^{*}=\frac{s_{i}^{-}}{S_{i}^{+}+S_{i}^{-}}
$$

Step 6: Rank the candidate Wi-Fi APs in descending order. The best Wi-Fi AP for offloading traffic will have the shortest distance to the positive ideal solution.

\section{B. Traffic Offloading using Wi-Fi}

In this section, we discuss an algorithm for traffic offloading from licensed band to Wi-Fi APs. Offloading initiates once a user enters within the range of $\mathrm{Wi}-\mathrm{Fi}$ APs indicating relatively higher signal strength than the cellular BS. Let ' $T_{r}$ ' be the time that a user resides within a coverage area of Wi$\mathrm{Fi}$, it can be approximately calculated with the direction of mobility and speed of the user. Let ' $B_{u}$ ' be the total amount of data in bytes to be transferred by the UE. The amount of data a user can offload, depends upon the residence time of a user in a selected Wi-Fi's cell. Let ' $b_{w}{ }^{\prime}$ and ' $b_{c}{ }^{\prime}$ be the bandwidth of Wi-Fi and cellular BS, respectively. The amount of data a user can offload through Wi-Fi of bandwidth ' $b_{w}{ }^{\prime}$ within a given time ' $T_{r}{ }^{\prime}$ is can be expressed as [32]:

$B_{w}=\left\{\begin{array}{c}\frac{b_{w} B_{u}}{b_{c}+b_{w}} \text { if } B_{u}>b_{w} T_{r} \\ b_{w} T_{r}, \text { otherwise }\end{array}\right\}$

From the above equation, it can be implied that if the data to be offloaded is greater than the Wi-Fi AP's transmission capability then it will be transmitted through the cellular network. The aforementioned can be formulated as,

$B_{w}=\frac{b_{w}}{b_{c}+b_{w}} P_{r}\left[B_{u}>b_{w} T_{r}\right]+b_{w} T_{r} P_{r}\left[B_{u} \leq b_{w} T_{r}\right]$

Using the procedure presented in [14], the above equation can be re-written as

$B_{w}=\frac{b_{w} B_{u}}{b_{c}+b_{w}}\left[1-R_{c}\left(\frac{B_{u}}{b_{w}}\right)\right]+b_{w} T_{r} \int_{0}^{B_{u}} / b_{w} T_{r} r_{c}\left(T_{r}\right) d T_{r}$
Where ' $R_{c}(.)^{\prime}$ is the distributed function of ' $T_{r}{ }^{\prime}$ and ' $1-R_{c}\left(\frac{B_{u}}{b_{w}}\right)^{\prime}$ represents the probability that the value of ${ }^{\prime}{\frac{B_{u}}{b_{w}}}^{\prime}$ is larger than the value of ${ }^{\prime} T_{r}{ }^{\prime}$.

\section{Algorithm Traffic Offloading}

1. Procedure

2. $T_{r}:$ Residence Time

3. $B_{u}$ : Total amount of data to be offloaded

4. $B_{w}$ : Amount of data offloaded through Wi-Fi

5. $B_{c}$ : Amount of data offloaded through Cellular BS

6. $S N R_{i}$ : SNR of userthe on each candidate Wi-Fi APs

7. $\delta_{i}$ : load on each candidate Wi-Fi APs

8. $b_{w}$ : bandwidth on each Wi-Fi APs

9. $R_{x}$ : received signal

10. Find the decision matrix $D$

11. Rank the candidate, and H-CRAN controller chooses the best candidate cell using the TOPSIS method as represented in equation 15 .

12. If $B_{u}<b_{w} T_{r}$ then

13. Offload all the data through selected Wi-Fi

14. Else

15. Send $B_{w}$ through Wi-Fi and $B_{c}=B_{u}-B_{w}$ through cellular BS

16. End

17. End Procedure

\section{Performance Analysis}

In this section, we analyze the performance of the proposed algorithm with the conventional Load balancing and SDN based Load balancing (LB) mechanisms. In SDN based load balancing algorithm, the decision is made by the SDN controller, based on optimized resource allocation [43]. It takes advantage of having a centralized controller that makes it a lot easier to find the optimal neighboring cell for load balancing. Hence unlike the traditional load balancing schemes, where the decision is usually based on received signal strength [44]. The QoS performance of the network is analyzed using metrics, such as cumulative handover, throughput, the extent of equilibrium \& offloading efficiency with respect to residence time and the number of Wi-Fi APs. The simulations have been carried out in MATLAB. Moreover, 37 hexagonal cells were considered with cellular BS at the center and each macrocell consists of 3 to 4 Wi-Fi AP's, which were distributed randomly within the geographical area of $1 \mathrm{~km}$. The Wi-Fi APs were placed in such a way that, there was no overlap between the Wi-Fi cells. Each user had a constant bit rate (CBR) of 1 Mbps. The bandwidth was assumed to be $10 \mathrm{MHz}$ with a capacity of 15 UEs in each macrocell. Rand walkway mobility was set for each user and having the mobility of $20 \mathrm{~m} / \mathrm{sec}$. Each user moved towards their pre-determined destination, and the positions were updated after every $1 \mathrm{sec}$. The long Logdistance path loss model was used as given in [45]. The transmitting power for the BS was set to be $46 \mathrm{dBm}$ as suggested in [46]. The antenna gain was set to be $7 \mathrm{~dB}$ and the Rayleigh fading model was designed to calculate the signal fading effects. The simulation parameters are also provided in Tab. 1.

The number of handovers is directly affected by the QoS of the network. Fig. 2 depicts the number of handovers for a specified simulation time to offload the data traffic. A smaller number of handovers are desired for better voice 
quality. Also with a higher number of handovers, the load on system significantly increases which inevitably results in reduced efficiency of the system [47]. The significant improvement in the handover with respect to simulation time can be understood by the fact that the handover is increased when the system consumes more time to offload the data from one network to another. Moreover, the proposed Rank based network selection algorithm has a smaller number of handovers compared to that of the other two algorithms. The proposed scheme reaches to 24 number of handovers in 50 seconds and goes up to a maximum of 150 handovers in 300 seconds, which is $30 \%$ and $50 \%$ less than the achieved number of handovers for SDN based LB and traditional LB, respectively. This is because the controller considers the knowledge of bandwidth availability, a load of neighboring cells, SNR and received signal strength. Based on all aforementioned metrics, a decision is made by the controller to handover the UE. Further, the SDN based load-balancing scheme takes into account the load on all neighboring cells while deciding for the handoff, but at times it could lead to improper handoffs in high-load scenarios and could also cause Ping-Pong effect.

Table 1. Simulation Parameters

\begin{tabular}{c|c}
\hline Parameter & Specification \\
\hline Number of Cell & 37 \\
Number of APs & $3-4$ \\
Cell layout & Hexagonal grid \\
Traffic Model & 1 Mbps $(\mathrm{CBR})$ \\
Cell radius & $150 \mathrm{~m}$ \\
Path loss & $-38.4-35.0 \log _{10} \mathrm{R}$ \\
System & $10 \mathrm{MHz}$ \\
Bandwidth & $20 \mathrm{~m} / \mathrm{s}$ \\
UE Movement & Rayleigh \\
Fading Model & $8 \mathrm{~dB}$ \\
Shadow fading & Lognormal with a standard deviation of \\
& $7 \mathrm{~dB}$ \\
Antenna Gain & $46 \mathrm{dBm}$ \\
eNodeB Tx & \\
Power &
\end{tabular}

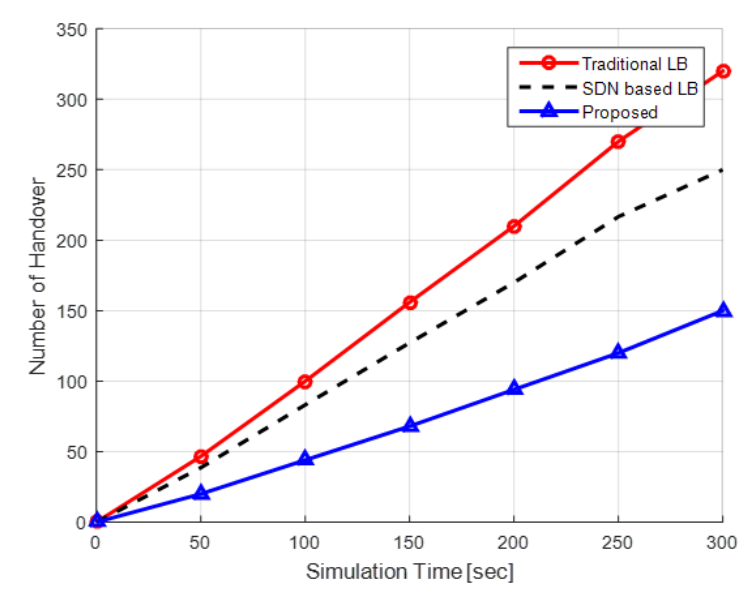

Fig. 2. Cumulative Handover

Fig. 3 shows the extent of load equilibrium within cells during the simulation period. It could be affected by simulation time because the load becomes more balanced in all the cells [48]. However, the proposed rank-based network selection algorithm outperforms the performance of traditional LB and SDN based LB schemes. The traditional
LB and SBN based LB approach have achieved $70 \%$ and $74 \%$, respectively, of the extent of load equilibrium. Though, when the simulation time reaches to a maximum of 80 seconds, the difference between the proposed scheme and the other two schemes is more visible. The proposed algorithm delivers around $94 \%$ of the extent of load equilibrium which is $5 \%$ and $12 \%$ higher than the SBN based LB and traditional LB approach. The reason is that load balancing is required mostly for the edge users, and unless the edge users find a Wi-Fi zone with the strong received signal, load balancing is not initiated. Since our proposed algorithm just not only consider the load of each candidate cell but also considers information related to other important parameters, hence it performs better than the other two algorithms.

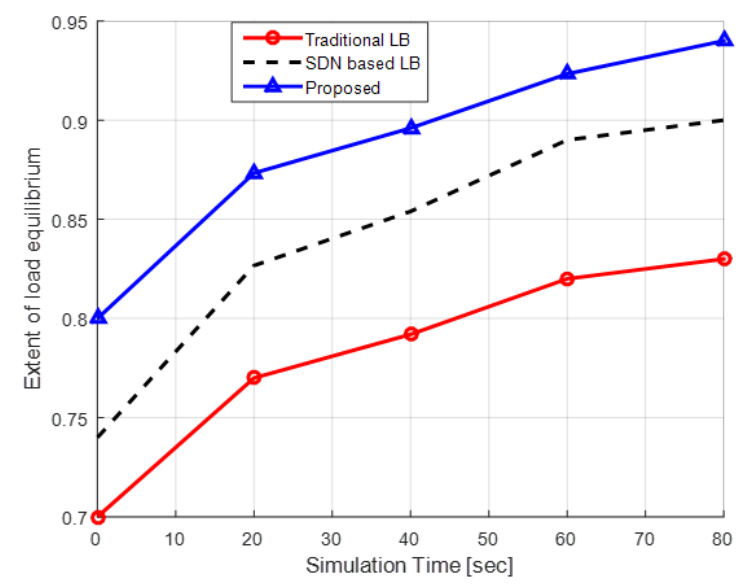

Fig. 3. Extent of Equilibrium

The performance of the proposed rank-based network selection algorithm can be further verified by the network throughput as shown in Fig. 4. From the figure, the number of bytes a user can make available at a particular time period to offload from the licensed band to the Wi-Fi band can be estimated. As also illustrated in Fig. 3, a greater extent of equilibrium will result in higher throughput, because of better and efficient resource utilization. Though, the noticeable decrement in the throughput after 40 seconds is because the network reaches its maximum peak value of the threshold. Resulting in network congestion and packet loss, which lowers the overall network throughput. The proposed technique is achieving around $13.8 \mathrm{Mbps}$ of throughput when the simulation is initiated, however, SDN based LB and traditional LB are delivering around 13.5 and $13 \mathrm{Mbps}$ throughput, respectively, at the start. Further, the proposed technique continued to achieve good throughput throughout the simulation time with a maximum of $14.2 \mathrm{Mbps}$ at the 40 second mark, which is $8 \%$ and $17 \%$ higher than the SDN based LB and traditional LB schemes, respectively.

Offloading efficiency is an important metric, which is given by total bytes transferred through the Wi-Fi network by the total bytes generated [49]. For simulation of offloading efficiency, each user takes input with specific arrival time and fixed size. The data file for uploading arrives at UE with a random inter-arrival time and random size, selected using the Weibull distribution that is, mean an as inter-arrival time and mean $b$ as file size. Hence, traffic can be calculated as b/a. Each file is scheduled for an upload in first input first output (FIFO) manner and the transmission time for each file is measured from the data rate of the user observed during the time of file transfer. If the transfer isn't 
completed within a residence time through the $\mathrm{Wi}-\mathrm{Fi}$ network, then the remaining data is uploaded through the $5 \mathrm{G}$ data network. The residence time of a user is varied, and the results are obtained as depicted in Fig. 5. It shows an offloading efficiency enhancement with an increase in residence time, which means more data is offloaded from the 5G network to a Wi-Fi network. To further validate this point, we also investigated the impact of Wi-Fi density on offloading efficiency. Fig. 6 shows the offloading efficiency in a cellular system related to the number of Wi-Fi APs in the same zone of communication. We kept the geographical area fixed to $1 \mathrm{~km}$ and then deployment of Wi-Fi APs in each cell was increased. In the simulation, we gradually increased the number of Wi-Fi AP's deployment from 1 to 6 without overlapping each other and then results were acquired. Results showed a considerable increase in offloading efficiency and almost reaches to $90 \%$ whereas the SDN based load balancing scheme took the efficiency form $50 \%$ to an efficiency of $85 \%$. On the other hand, with an increase in the number of Wi-Fi APs, the proposed model improves the efficiency of data offloading, now system initializes with $60 \%$ efficiency, means an improved accuracy of $5 \%$ in the initial phase and so on, based on the number of the Wi-Fi APs.

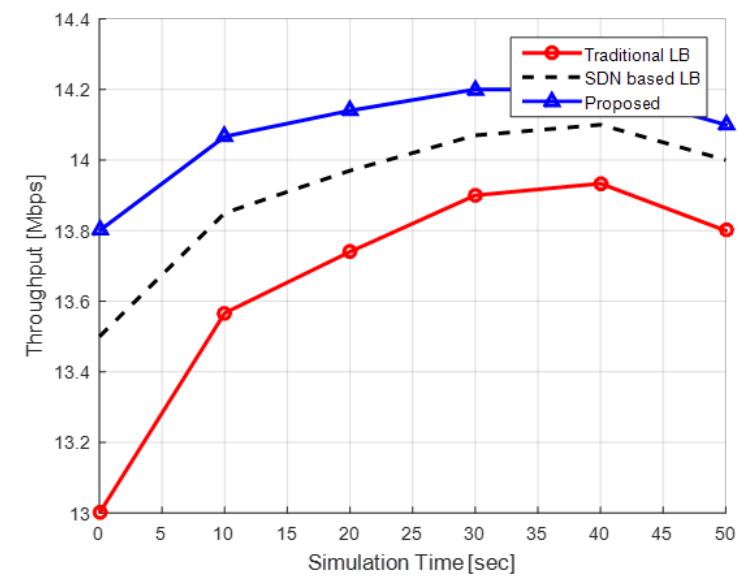

Fig. 4. Network Throughput

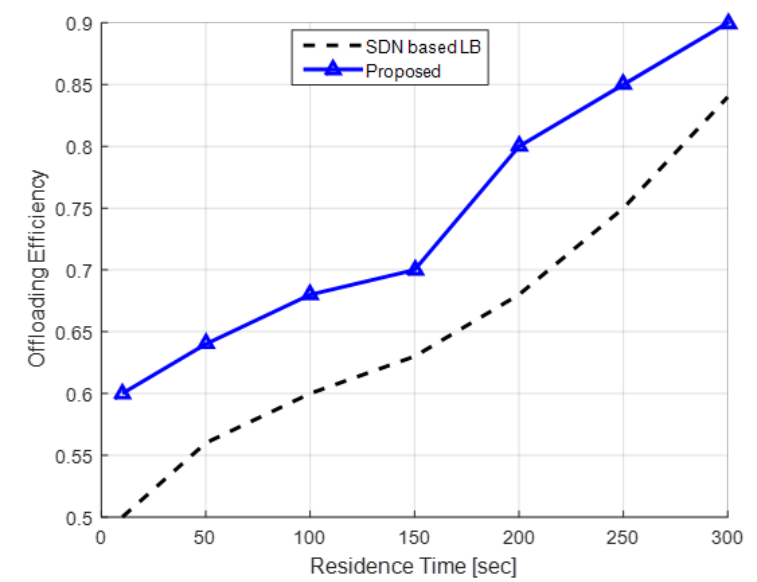

Fig. 5. Offloading Efficiency vs Residence Time

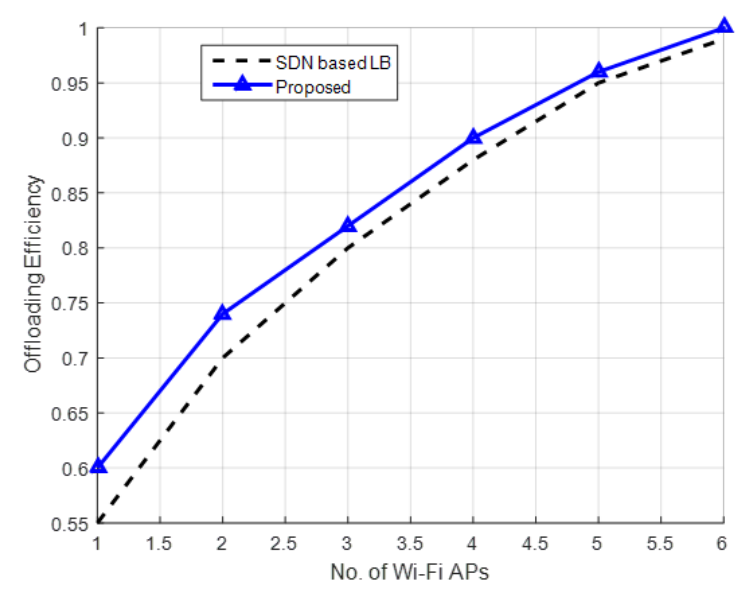

Fig. 6. Offloading Efficiency vs Number of Wi-Fi AP's

\section{Conclusion}

Rapid growth in user-end applications leads to an exponential increase in data traffic generation, resulting in massive overloading of cellular networks. In this work, we propose a simple opportunistic model to offload data traffic to already existing Wi-Fi networks with proper network selection scheme, in case a Wi-Fi link is available within the range. The network selection is made using the TOPSIS algorithm, in which SNR, received signal strength, network load, and available bandwidth are taken as benefit parameters. The network with maximum rank is selected as the candidate network for data offloading. The amount of data offloaded within a network depends upon the residence time. If a user is unable to offload the whole amount of data within its stay duration in a Wi-Fi range, the remaining amount of data is offloaded through the $5 \mathrm{G}$ data network. The performance was then analyzed based on cumulative handover, throughput, extent of equilibrium and offload efficiency. The simulation results showed that the proposed rank-based network selection scheme outperforms the traditional LB and SDN based LB mechanisms. Since, network selection just not only based on receive signal strength, hence a user in H-CRAN setup gets a Wi-Fi AP link, which provides better throughput and high residence time. Further, in actual terms, the offloading efficiency depends upon the amount of data offloaded to the total amount of data, so an increased residence time and Wi-Fi density will eventually lower the load on the $5 \mathrm{G}$ cellular network.

This is an Open Access article distributed under the terms of the Creative Commons Attribution License

\section{References}

[1] T. Abbas, F. Qamar, I. Ahmed, K. Dimyati, and M. B. Majed, "Propagation channel characterization for 28 and $73 \mathrm{GHz}$ millimeter-wave 5G frequency band," in Research and
Development (SCOReD), 2017 IEEE 15th Student Conference on, 2017, pp. 297-302: IEEE. 


\section{Journal of Engineering Science and Technology Review 12 (2) (2019) 9 - 16}

[2] F. Qamar, M. H. S. Siddiqui, K. Dimyati, K. A. B. Noordin, and M. B. Majed, "Channel characterization of 28 and $38 \mathrm{GHz}$ MM-wave frequency band spectrum for the future $5 \mathrm{G}$ network," in Research and Development (SCOReD), 2017 IEEE 15th Student Conference on, 2017, pp. 291-296: IEEE.

[3] F. Qamar, T. Abbas, M. N. Hindia, K. B. Dimyati, K. A. B. Noordin, and I. Ahmed, "Characterization of MIMO propagation channel at $15 \mathrm{GHz}$ for the $5 \mathrm{G}$ spectrum," in Communications (MICC), 2017 IEEE 13th Malaysia International Conference on, 2017, pp. 265-270: IEEE.

[4] M. N. Tehrani, M. Uysal, and H. Yanikomeroglu, "Device-todevice communication in $5 \mathrm{G}$ cellular networks: challenges, solutions, and future directions," IEEE Communications Magazine, vol. 52, no. 5, pp. 86-92, 2014.

[5] M. N. Hindia, F. Qamar, T. A. Rahman, and I. S. Amiri, "A Stochastic Geometrical Approach for Full-Duplex MIMO Relaying Model of High-Density Network," Ad Hoc Networks, 2018.

[6] X. Lijun and L. Chunlin, "Dynamic Service Provisioning and Selection for Satisfying Cloud Applications and Cloud Providers in Hybrid Cloud," International Journal of Cooperative Information Systems, vol. 26, no. 04, p. 1750005, 2017.

[7] F. Qamar, K. B. Dimyati, M. N. Hindia, K. A. B. Noordin, and A. M. Al-Samman, "A comprehensive review on coordinated multipoint operation for LTE-A," Computer Networks, vol. 123, pp. 1937, 2017.

[8] O. Elijah, T. A. Rahman, I. Orikumhi, C. Y. Leow, and M. N. Hindia, "An Overview of Internet of Things (IoT) and Data Analytics in Agriculture: Benefits and Challenges," IEEE Internet of Things Journal, 2018.

[9] H. R. Chayon, K. Dimyati, H. Ramiah, and A. W. Reza, "An Improved Radio Resource Management with Carrier Aggregation in LTE Advanced," Applied Sciences, vol. 7, no. 4, p. 394, 2017.

[10]M. Shikh-Bahaei, Y.-S. Choi, and D. Hong, "Full-Duplex and Cognitive Radio Networking for the Emerging 5G Systems," Wireless Communications and Mobile Computing, vol. 2018, 2018.

[11]D. Udeshi and F. Qamar, "Quality Analysis Of Epon Network For Uplink and Downlink Design," Asian Journal of Engineering, Sciences \& Technology, vol. 4, no. 2, 2014.

[12]J. Zhang, L. Dai, X. Li, Y. Liu, and L. Hanzo, "On low-resolution ADCs in practical $5 \mathrm{G}$ millimeter-wave massive MIMO systems," IEEE Communications Magazine, 2018.

[13] M. Liaqat, K. A. Noordin, T. A. Latef, and K. Dimyati, "Powerdomain non orthogonal multiple access (PD-NOMA) in cooperative networks: an overview," Wireless Networks, pp. 1-23, 2018

[14] M. Conti and S. Giordano, "Mobile ad hoc networking: milestones, challenges, and new research directions," IEEE Communications Magazine, vol. 52, no. 1, pp. 85-96, 2014.

[15]T. Wang, Y. Zhou, X. Wang, and Y. Cao, "A social-based DTN routing in cooperative vehicular sensor networks," International Journal of Cooperative Information Systems, vol. 27, no. 01, p. 1741003, 2018.

[16]C. Li, J. Zhang, and Y. Chen, "Media Cloud Service Scheduling Optimization for Resource-Intensive Mobile Application," International Journal of Cooperative Information Systems, vol. 27, no. 04, p. 1850008, 2018.

[17]A. Gachhadar, M. N. Hindia, F. Qamar, M. H. S. Siddiqui, K. A. Noordin, and I. S. Amiri, "Modified genetic algorithm based power allocation scheme for amplify-and-forward cooperative relay network," Computers \& Electrical Engineering, 2018.

[18]K. A. B. Noordin, M. N. Hindia, F. Qamar, and K. Dimyati, "Power Allocation Scheme Using PSO for Amplify and Forward Cooperative Relaying Network," in Science and Information Conference, 2018, pp. 636-647: Springer.

[19] M. N. Hindia, F. Qamar, M. B. Majed, T. A. Rahman, and I. S. Amiri, "Enabling remote-control for the power sub-stations over LTE-A networks," Telecommunication Systems, pp. 1-17, 2018.

[20]W. OBILE, "Ericsson Mobility Report," ed: Nov, 2016.

[21] M. Peng, Y. Li, J. Jiang, J. Li, and C. Wang, "Heterogeneous cloud radio access networks: A new perspective for enhancing spectral and energy efficiencies," IEEE Wireless Communications, vol. 21, no. 6, pp. 126-135, 2014.

[22]F. Qamar, K. B. Dimyati, M. N. Hindia, K. A. B. Noordin, and A. M. Al-Samman, "A Comprehensive Review on Coordinated MultiPoint Operation for LTE-A," Computer Networks, 2017.

[23]B. Han, P. Hui, V. A. Kumar, M. V. Marathe, J. Shao, and A. Srinivasan, "Mobile data offloading through opportunistic communications and social participation," IEEE Transactions on Mobile Computing, vol. 11, no. 5, pp. 821-834, 2012.
[24]K. Lee, J. Lee, Y. Yi, I. Rhee, and S. Chong, "Mobile data offloading: How much can WiFi deliver?," in Proceedings of the 6th International COnference, 2010, p. 26: ACM.

[25]D. Liu, L. Khoukhi, and A. Hafid, "Data offloading in mobile cloud computing: A Markov Decision Process approach," in Communications (ICC), 2017 IEEE International Conference on, 2017, pp. 1-6: IEEE.

[26]A. Ravichandran, A. Alnoman, N. Sharma, and A. Anpalagan, "Traffic offloading problem in two-tier HetNets with D2D support for emergency communications," in Humanitarian Technology Conference (IHTC), 2017 IEEE Canada International, 2017, pp. 128-132: IEEE.

[27] J. Du, E. Gelenbe, C. Jiang, H. Zhang, and Y. Ren, "Contract Design for Traffic Offloading and Resource Allocation in Heterogeneous Ultra-Dense Networks," IEEE Journal on Selected Areas in Communications, 2017.

[28] W. Hu and G. Cao, "Quality-aware traffic offloading in wireless networks," IEEE Transactions on Mobile Computing, 2017.

[29] S. Kabiri, H. Kalbkhani, T. Lotfollahzadeh, M. G. Shayesteh, and V. Solouk, "Technique for order of preference by similarity to ideal solution based predictive handoff for heterogeneous networks," IET Communications, vol. 10, no. 13, pp. 1682-1690, 2016.

[30]X. Duan, A. M. Akhtar, and X. Wang, "Software-defined networking-based resource management: data offloading with load balancing in 5G HetNet," EURASIP Journal on Wireless Communications and Networking, vol. 2015, no. 1, pp. 1-13, 2015.

[31]G. S. Aujla, R. Chaudhary, N. Kumar, J. J. Rodrigues, and A. Vinel, "Data Offloading in 5G-Enabled Software-Defined Vehicular Networks: A Stackelberg-Game-Based Approach," IEEE Communications Magazine, vol. 55, no. 8, pp. 100-108, 2017.

[32] J.-L. Chen, Y.-W. Ma, H.-Y. Kuo, C.-S. Yang, and W.-C. Hung, "Software-Defined Network Virtualization Platform for Enterprise Network Resource Management," IEEE Transactions on Emerging Topics in Computing, vol. 4, no. 2, pp. 179-186, 2016.

[33] Y.-S. Chen, C.-S. Hsu, T.-Y. Juang, and H.-H. Lin, "An energyaware data offloading scheme in cloud radio access networks," in Wireless Communications and Networking Conference (WCNC), 2015 IEEE, 2015, pp. 1984-1989: IEEE.

[34] J. Wu, J. Liu, Z. Huang, C. Du, H. Zhao, and Y. Bai, "Intelligent network selection for data offloading in $5 \mathrm{G}$ multi-radio heterogeneous networks," China Communications, vol. 12, no. Supplement, pp. 132-139, 2015.

[35]E. Fakhfakh and S. Hamouda, "Incentive reward for efficient WiFi offloading using Q-learning approach," in Wireless Communications and Mobile Computing Conference (IWCMC), 2017 13th International, 2017, pp. 1114-1119: IEEE.

[36] M. Peng, Y. Li, Z. Zhao, and C. Wang, "System architecture and key technologies for $5 \mathrm{G}$ heterogeneous cloud radio access networks," IEEE network, vol. 29, no. 2, pp. 6-14, 2015.

[37] I. Broustis, K. Papagiannaki, S. V. Krishnamurthy, M. Faloutsos, and V. P. Mhatre, "Measurement-driven guidelines for 802.11 WLAN design," IEEE/ACM Transactions on Networking (TON), vol. 18, no. 3, pp. 722-735, 2010.

[38]H. Velayos, V. Aleo, and G. Karlsson, "Load balancing in overlapping wireless LAN cells," in Communications, 2004 IEEE International Conference on, 2004, vol. 7, pp. 3833-3836: IEEE.

[39]G. Wu and T.-c. Chiueh, "Passive and accurate traffic load estimation for infrastructure-mode wireless lan," in Proceedings of the 10th ACM Symposium on Modeling, analysis, and simulation of wireless and mobile systems, 2007, pp. 109-116: ACM.

[40] V. P. Mhatre and K. Papagiannaki, "Optimal design of high density 802.11 WLANs," in Proceedings of the 2006 ACM CoNEXT conference, 2006, p. 8: ACM.

[41]G. Bianchi, "Performance analysis of the IEEE 802.11 distributed coordination function," IEEE Journal on selected areas in communications, vol. 18, no. 3, pp. 535-547, 2000.

[42]L. Anojkumar, M. Ilangkumaran, and V. Sasirekha, "Comparative analysis of MCDM methods for pipe material selection in sugar industry," Expert Systems with Applications, vol. 41, no. 6, pp. 2964-2980, 2014

[43] S. Namal, I. Ahmad, A. Gurtov, and M. Ylianttila, "SDN based inter-technology load balancing leveraged by flow admission control," in Future Networks and Services (SDN4FNS), 2013 IEEE SDN for, 2013, pp. 1-5: IEEE.

[44]L. MacVittie, "Message-Based Load Balancing," Technical Brief, pp. 1-9, 2010.

[45]T. R. Port, "Wireless Communications Principle and Practice Prentice Hall PTR upper Saddle River," New Jersey, vol. 758, no. 1996, pp. 45-48, 1996. 
Anand Gachhadar, Faizan Qamar, Dhawa Sang Dong, Mohammed B. Majed, Effariza Hanafi and Iraj Sadegh Amiri/

$$
\text { Journal of Engineering Science and Technology Review } 12 \text { (2) (2019) } 9 \text { - } 16
$$

[46]T. ETSI, "125 211 V3. 1.1 Universal Mobile Telecommunications System (UMTS)," Physical channels and mapping of transport channels onto physical channels (FDD)(3GPP TS 25.211 version 6.1. 0 Release 6), pp. 0000-0001, 2000.

[47] M. N. Hindia, A. W. Reza, and K. A. Noordin, "Investigation of a New Handover Approach in LTE and WIMAX," The Scientific World Journal, vol. 2014, 2014.

[48]X. Duan, X. Wang, and A. M. Akhtar, "Partial mobile data offloading with load balancing in heterogeneous cellular networks

using software-defined networking," in Personal, Indoor, and Mobile Radio Communication (PIMRC), 2014 IEEE 25th Annual International Symposium on, 2014, pp. 1348-1353: IEEE.

[49]D. Hinger and D. Kalbande, "Review of mobile data offloading through Wi-Fi," in Circuits, Systems, Communication and Information Technology Applications (CSCITA), 2014 International Conference on, 2014, pp. 425-429: IEEE. 